\title{
Follow-Up Study of Electrocardiographic Indices in 252 Children with Ventricular Septal Defect after Minimally Invasive Transthoracic Closure
}

\author{
Yanan Sun, Minglei Gao, Ping Wen \\ Dalian Women and Children's Medical Group, Dalian, Liaoning Province, China
}

\section{ABSTRACT}

Objective: The aim of this study was to explore the efficacy and safety of minimally invasive transthoracic closure (MITC) in treating ventricular septal defect (VSD).

Methods: A total of 252 children with VSD were investigated between August 2013 and March 2015. Their electrocardiographic indices were monitored at different time points (before surgery [T0], immediately after surgery [T1], and 3 days/ 7 days $/ 1$ month $/ 3$ months/ 6 months after surgery [T2-6]) and followed up every year. Related adverse events also were recorded.

Results: All children with VSD successfully underwent MITC. The average follow-up time was 5 years, during which no changes in the heart rate or corrected QT interval were found. The PR and QRS intervals were prolonged in the early postoperative period (T3); the SV1+RV5 amplitude decreased significantly at T6; and the left axis deviation significantly recovered at T6 $(P<0.05)$. A total of 32 patients developed arrhythmia within 3 days after surgery; however, no severe arrhythmia, including ventricular tachycardia, ventricular fibrillation, second- and third-degree atrioventricular block, complete left or right bundle branch block, and delayed arrhythmia, occurred during the follow-up period. Binary multivariate logistic regression revealed that the longer the surgery time and the larger the VSD diameter, the higher the risk of arrhythmia.

Conclusions: The incidence of long-term arrhythmia after MITC is low, and the outcomes are good.

\section{INTRODUCTION}

Ventricular septal defects (VSDs), which are holes between the ventricles, are recognized as the most common congenital cardiac malformation, accounting for almost $50 \%$ of all congenital heart diseases [Cho 2017] and can exist in isolation. They can be complicated by additional intracardiac lesions or can be part of more complex combinations [Soto 1980; Faccini 2019].

Received fune 1, 2021; accepted October 11, 2021.

Correspondence: Ping Wen, Dalian Women and Children's Medical Group, 154 Zhongshan Road, Xigang District, Dalian 116012, Liaoning Province, China, Telephone+8613504115999 (e-mail: pwdocn@163.com).
Accurately estimating the prevalence of VSD is difficult [Spicer 2014]. The estimated prevalence is 2 to 3.94 per 1000 live births, as diagnosed by echocardiography [Penny 2011; Li 2016]. In some studies, the incidence of isolated VSD is $1.25 \%$. Most cases are closed naturally; in cases where they are not closed and are sufficiently small to restrict shunting to the extent that there are no symptoms, additional palliative measures are unnecessary [Gerbode 1958], and only $0.02 \%$ need surgical treatment [Cho 2017].

Clinical management of isolated VSD is imperative because the long-term persistence of such a defect may cause complications [Habib 2015]. In patients referred for surgical correction, the defects are almost always closed by directly placing a patch from the right ventricular side [Spicer 2013]. Traditionally, open-heart surgical repair with midline sternotomy and cardiopulmonary bypass (CPB) has been the mainstay of therapy for many years [Rein 1977], and catheterbased intervention has been initially introduced for the closure of muscular VSD. Transesophageal ultrasound-guided minimally invasive transthoracic closure (MITC) has become increasingly mature in recent years and gradually has become a new surgical method for the treatment of VSD [Liang 2017; Ou-yang 2017]. This technology has the advantages of simple surgery, no extracorporeal circulation, no limitation of patient age and body mass, wide indications, short operating distance, and avoidance of radiation [Zeng 2008; Xing 2009; Zhang 2013]. With the extensive development of this technology, the clinical prognosis has been affirmed. However, there still is a lack of long-term follow-up data, together with arrhythmia as the most common complication after VSD. Thus, follow up of electrocardiographic (ECG) indices is necessary. ECG indices can directly reflect the electrophysiological situation of each part of the heart, objectively record the changes on ECG, indirectly reflect the hemodynamic changes, and evaluate the direct influence of the surgery on the heart. VSD is closely related to the anatomy of the heart conduction tract, which may cause arrhythmias, such as atrioventricular block and bundle branch block after surgery. In this study, a total of 252 children who underwent MITC for VSD were selected for follow-up analysis, aiming to explore the changes in the ECG indices and outcomes postoperatively.

\section{MATERIALS AND METHODS}

Subjects: A total of 252 patients with VSD (118 boys and 134 girls aged 5-130 [22.8 \pm 25.0$]$ months, with a body weight 
of 5.5-95 [12.1 \pm 8.4$] \mathrm{kg}$ ), who underwent MITC in our center from August 2013 to March 2015 were selected. Transthoracic echocardiography showed that the VSD diameter was 2-10 (3.4 \pm 1.4$) \mathrm{mm}$. According to Anderson's classification system, the types of VSD included perimembranous VSD $(\mathrm{N}=231 ; 91.7 \%)$ and doubly committed subarterial VSD $(\mathrm{N}=21 ; 8.3 \%)$. According to the Chinese Expert Consensus of Transthoracic Minimally Invasive Closure for Ventricular Defect [Cardiovascular Surgeons Branch of Chinese Medical Association 2011], the indications of surgery included the following: 1) age of $\geq 3$ months, 2) simple perimembranous VSD with hemodynamic abnormalities and VSD diameter of 4-8 mm within 1 year of age, 3) simple muscular VSD with hemodynamic abnormalities and VSD diameter of $>3 \mathrm{~mm}$ and multiple muscular VSD, 4) subpulmonic VSD without obvious aortic valve prolapse and with VSD diameter of $<6 \mathrm{~mm}$ within 1 year of age, 5) residual shunt after surgery, and 6) myocardial infarction and posterior traumatic ventricular septal perforation. The family members of the patients signed the surgical informed consent form, and the study was approved by the ethics committee of our hospital.

Methods: The surgical method was performed in accordance with the Chinese Expert Consensus of Transthoracic Minimally Invasive Closure for Ventricular Defect issued in

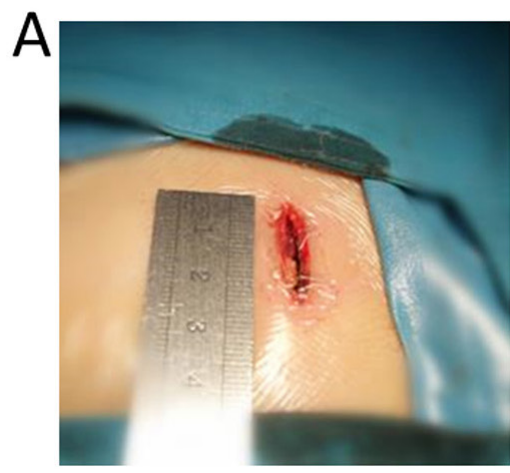

B

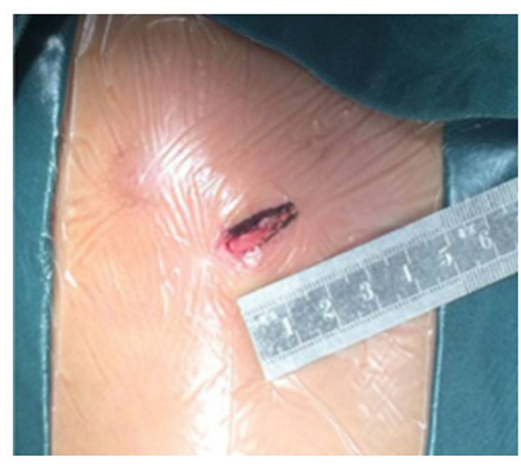

E

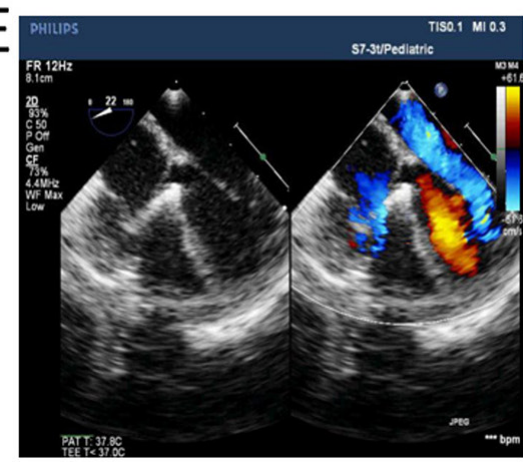

$\mathrm{H}$

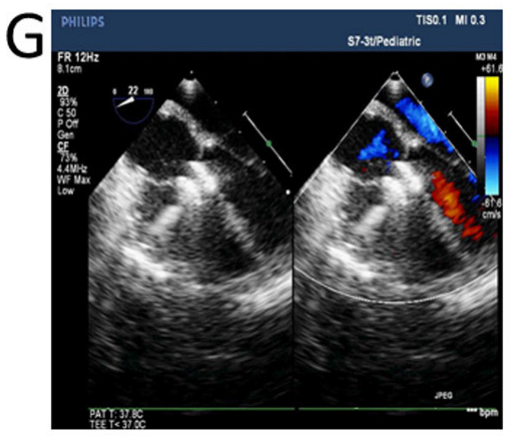

D

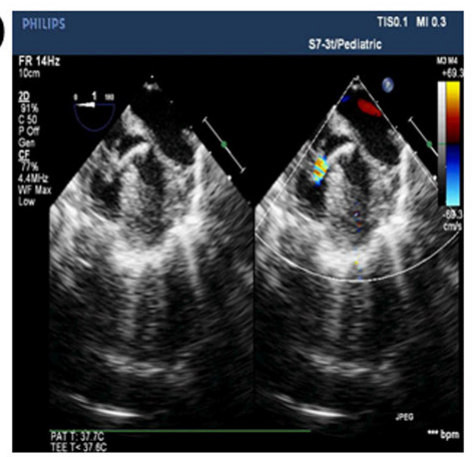

PArisice

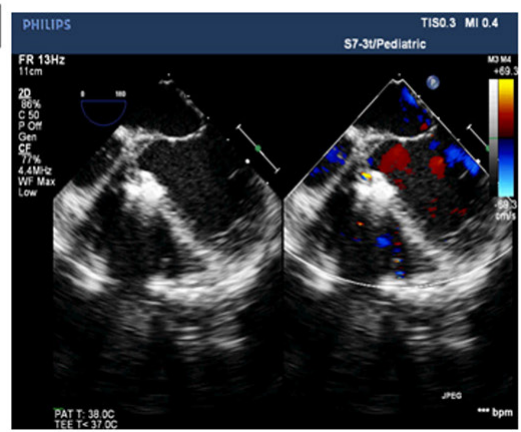

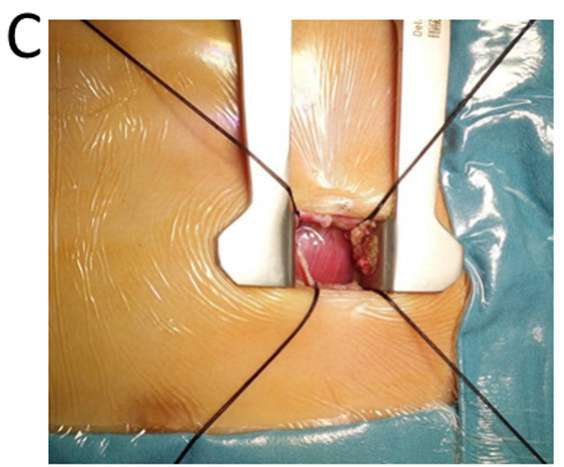

$\mathrm{F}$
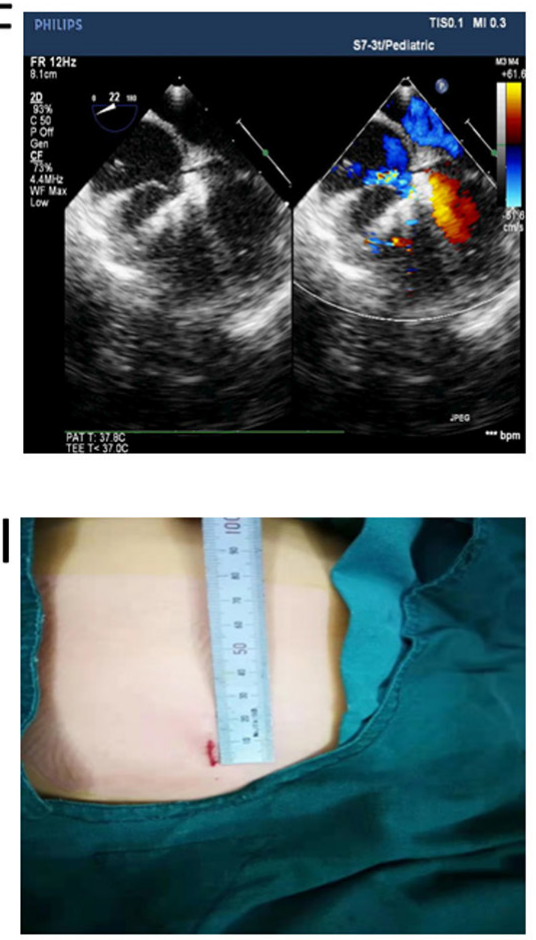

Figure 1. Photos of the procedure in the study. Choose different surgical incisions, according to the position of the VSD. A) Small longitudinal incision at the lower end of the sternum. B) The third intercostal transverse incision on the left edge of the sternum.) C) Cut a small part of the pericardium to expose the free wall of the right ventricle, determine the puncture site of the free wall of the right ventricle, and use a mattress or purse-string suture. D) Establish the delivery track: puncture and insert the guide wire under the guidance of esophageal ultrasound (TEE) and enter the left ventricular cavity through VSD. E) The delivery sheath is inserted along the guide wire and reaches the left ventricular cavity through the VSD. The occluder is slowly pushed out under realtime TEE monitoring. F) Release the left ventricular umbrella of the occlude. G) Release the right ventricular umbrella of the occlude. $H$ ) TEE fully evaluates, confirms that everything is normal, and finally releases the occlude. I) Postoperative incision. 
2011 [Cardiovascular Surgeons Branch of Chinese Medical Association 2011] and under general anesthesia with tracheal intubation. The incision site depended on the type of the defect. The lower end of the sternum or the lower part of the sword process was selected for the perimembranous defects. Meanwhile, the left edge of the sternum was selected for the subarterial defects; a small transverse incision was created at the third intercostal space, and the length was approximately $2-2.5 \mathrm{~cm}$. The pericardium was cut into small parts, and the free wall of the right ventricle was exposed. Heparin was injected to the vein at a dose of $100 \mu / \mathrm{kg}$. The appropriate puncture site was selected under the guidance of TEE: Avoid the coronary artery; press fingers on the front wall of the right ventricle; confirm the puncture site with a TEE image; and suture with a gasket or bag for one needle. After the right ventricular anterior wall was punctured by the artery cannula, the needle core was withdrawn and sent to the soft guidewire. The guidewire was led by TEE through the VSD and into the left ventricular cavity. The outer sheath of the puncture needle was exited, and the dilated sheath tube and the delivery sheath tube were placed along the guidewire (the expanding sheath tube was the inner core of the sheath tube); the left ventricular cavity was reached through the VSD, and the transport track was established. After the delivery sheath tube was placed in the left ventricle, the guidewire was withdrawn; the expanding sheath tube was kept in the left ventricle; and the outer end of the sheath tube was connected to the loading sheath tube with the blocking device. Under real-time monitoring of TEE, the sealing device was pushed slowly, and the left disk surface was released. The entire sheath was then withdrawn to make the left disc face close to the left ventricular surface of the compartment interval, and immediately carefully detected the shunt, whether there was an atrioventricular valve activity disorder, whether the surrounding tissue was embedded; no abnormal condition, gently withdrawn sheath tube, and release of the waist and right pan of the blocker. TEE multi-section observation of the position and shape of the occluder, repeated push-andpull test, if there is no displacement, no residual shunt, and valve reflux, ECG indicates no serious conduction block. The blocker can be released; the conveyor can be withdrawn; and the suture knot and surgery can be completed to achieve successful blocking treatment. (Figure 1) Protamine was administered after heparin injection $(1: 1)$ and aspirin 6 hours after surgery. In all 252 cases, VSD occluders with a diameter of 4-12 $(5.4 \pm 1.4) \mathrm{mm}$ were inserted. The operation time was 30-155 $(68.1 \pm 24.8) \mathrm{min}$. The occluders were selected from Shenzhen Xianjian Technology Co., Ltd.; Beijing Huayi Shengjie Co.; Shanghai Shape Memory Co., Ltd.; and AGA Co., Ltd., US. Symmetrical or eccentric occluders were selected based on individual transthoracic cardiac ultrasound findings.

Follow up: Aspirin was administered orally at a dose of $3-5 \mathrm{mg} / \mathrm{kg} \cdot \mathrm{d}$ for 6 months. After surgery, there were 13 cases of new micro to mild tricuspid regurgitation, three cases of pericardial effusion, which were resolved after pericardiocentesis, and one case of perimembranous VSD with a micro residual shunt, which closed spontaneously after 3 months. The follow-up period was 5 years. The 12-lead ECG indices were monitored at several time points, namely, before surgery (T0), immediately after surgery (T1), on days 3 and 7 after surgery (T2 and $\mathrm{T} 3$, respectively), and at months 1,3 , and 6 after surgery (T4, T5, and T6, respectively). All ECG indices were entered into one computer for storage (various indices along ECG information management system, 2013), which were automatically calculated as the computer and a professional proofread the heart rate, PR interval, QRS interval, corrected QT interval (QTc), and SV1+RV5 amplitude. The electric axis, rhythm, QRS shape, and ST-T changes were also observed, together with the occurrence of palpitation, syncope, and dark haze.

Statistical analysis: The SPSS software (version 23.0) was used for statistical processing. The measurement data were expressed as means \pm SDs. The normally distributed data were compared using one-way ANOVA and the skewed-distributed data using the non-parametric test. The count data were expressed as the composition ratio and analyzed using the chi-square test. The occurrence of complications, such as arrhythmia, during follow up was set as the subgrouping factor for the binary multivariate logistic regression. P-values of $<0.05$ were considered statistically significant.

\section{RESULTS}

All children with VSD were followed up for 5.0-7.5 $(5.7 \pm 0.6)$ years for comparing the various indices, cardiac functions, and cardiac structural features. (Table 1) The heart rate, PR interval, QRS interval, QTc, and SV1+RV5 amplitude at the various postoperative time points were compared with those at the preoperative time point. There was no significant difference found in the heart rate and QTc; the PR interval was significantly prolonged at T1-3 $(P<0.05)$; the QRS interval was significantly prolonged at T2 and T3 $(P<$ $0.05)$; the SV1+RV5 amplitude significantly decreased at T6 $(P<0.05)$; and the left axis deviation significantly recovered at T6 $(P<0.05)$. (Table 2)

Postoperative arrhythmia occurred concentratedly 3 days after surgery, with a total of 32 cases $(12.7 \%)$, including five cases of first-degree atrioventricular block (with the criterion shown in the supplementary table), which returned to normal 1 year after surgery; two cases of complete right bundle branch block (CRBBB; children: $\geq 0.10$ s, adults: $\geq 0.12 \mathrm{~s}$ ), which converted to incomplete right bundle branch block (IRBBB) 1 and 2 years after surgery, respectively, and did not return to normal while showing no clinical symptoms at 5 years after surgery; and 16 cases of IRBBB (children: $0.08-0.10$ s, adults: $0.08-0.12 \mathrm{~s}$ ), which recovered 3 years after surgery. No severe or delayed arrhythmias occurred during follow up. (Table 3 )

With the occurrence of postoperative arrhythmia as a subgrouping factor, the differences between the groups were analyzed. (Table 4) The factors with significant differences in the univariate analysis were included in the binary multivariate logistic regression analysis, and the likelihood 
ratio forward method was used. The inclusion criterion was 0.05 , and the exclusion criterion was 0.10 . Among the factors, the operation time $(77.83 \pm 23.37 \mathrm{~min})$, VSD diameter $(4.28 \pm 1.92 \mathrm{~mm})$, and occluder model $(6.50 \pm 1.66 \mathrm{~mm})$ were found as the risk factors (OR value) for postoperative arrhythmia $(\mathrm{OR}>1)$. Conversely, the presence of a membranous tumor was found as a protective factor for postoperative arrhythmia $(\mathrm{OR}<1)$; the children without membranous tumors were more prone to experiencing arrhythmia after surgery. (Table 5)

\section{DISCUSSION}

Owing to the close anatomical relationship between VSD and the conduction bundle in the area of the peripheral membrane, as well as the His bundle and its left/right bundle branches running on the posterior and inferior edges of the VSD (approximately 2-4 $\mathrm{mm}$ from the edge of the VSD), arrhythmia is the most common complication after the development of VSD regardless of extracorporeal repair or surgical closure.

Table 1. ECG follow-up data of VSD patients after transthoracic minimally invasive occlusion

\begin{tabular}{lcccc}
\hline Time point & LVEF & LVFS & LAD & LVEDD \\
\hline T0 & $65.49 \pm 3.54$ & $36.39 \pm 2.24$ & $19.90 \pm 2.12$ & $34.36 \pm 5.66$ \\
T1 & $63.22 \pm 2.12$ & $34.65 \pm 3.54$ & $19.57 \pm 2.83$ & $33.94 \pm 4.95$ \\
T2 & $63.25 \pm 3.82$ & $34.86 \pm 2.46$ & $19.43 \pm 4.24$ & $33.91 \pm 4.83$ \\
T3 & $63.92 \pm 1.41$ & $34.30 \pm 3.41$ & $19.64 \pm 2.47$ & $33.86 \pm 3.41$ \\
T4 & $63.29 \pm 1.41$ & $34.72 \pm 2.71$ & $18.81 \pm 3.54$ & $33.50 \pm 5.45$ \\
T5 & $64.17 \pm 2.12$ & $34.63 \pm 3.54$ & $18.02 \pm 3.17$ & $32.86 \pm 3.72$ \\
T6 & $64.10 \pm 0.71$ & $35.05 \pm 3.55$ & $17.38 \pm 3.21$ & $31.90 \pm 3.83$ \\
1 year after surgery & $65.37 \pm 1.41$ & $35.10 \pm 2.58$ & $16.96 \pm 2.75$ & $30.11 \pm 3.54 *$ \\
2 years after surgery & $65.44 \pm 4.24$ & $35.54 \pm 3.14$ & $16.77 \pm 2.71$ & $30.24 \pm 4.78^{*}$ \\
3 years after surgery & $65.78 \pm 2.12$ & $36.12 \pm 2.16$ & $16.63 \pm 1.41$ & $29.96 \pm 5.39 *$ \\
4 years after surgery & $65.58 \pm 3.54$ & $36.51 \pm 2.83$ & $16.56 \pm 2.74$ & $29.79 \pm 4.04^{*}$ \\
5 years after surgery & $65.72 \pm 3.72$ & $36.38 \pm 2.12$ & $16.45 \pm 3.56$ & $29.65 \pm 3.76^{*}$ \\
\hline
\end{tabular}

Compare with T0, $* P<0.05$

Table 2. Follow-up data of ECG after MITC

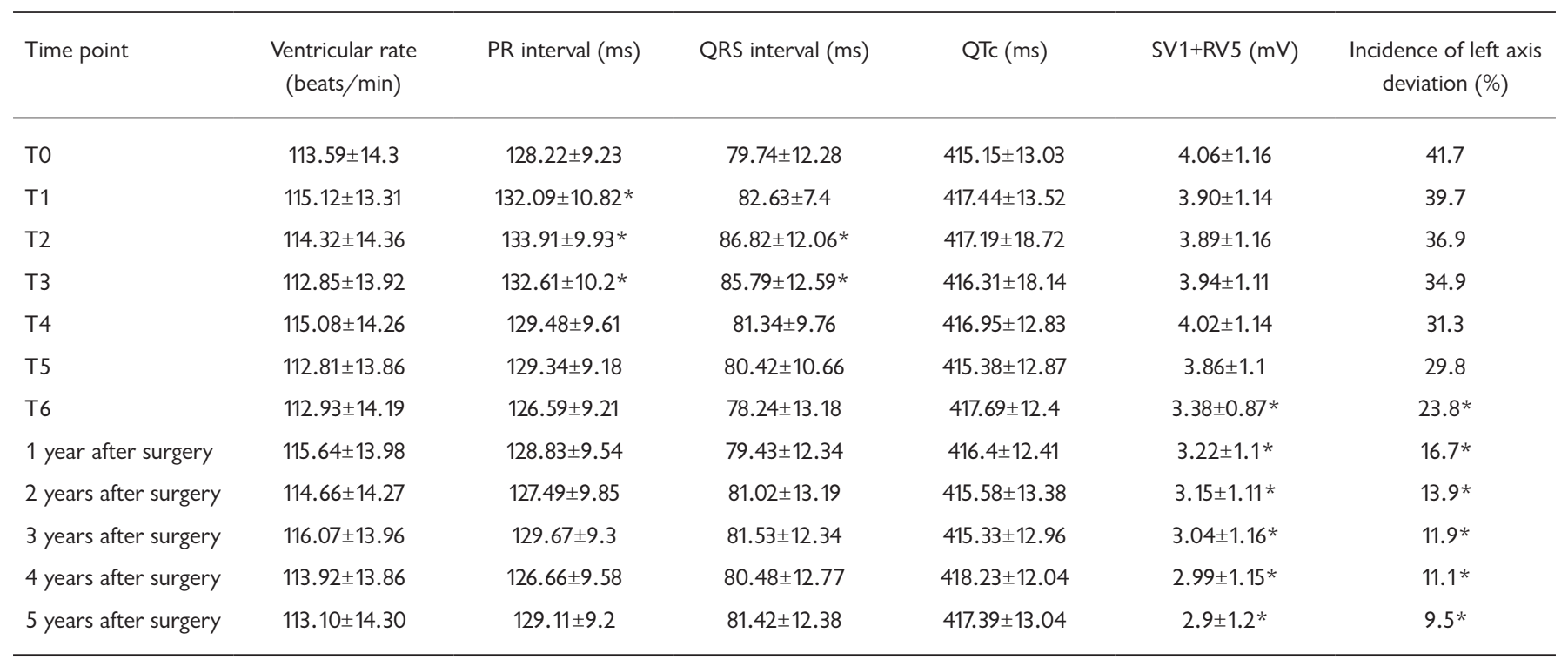

Compared with the preoperative data, ${ }^{*} P<0.05$ 
In China and abroad, follow-up studies on the changes in ECG indices after VSD development mostly have focused on VSD repair and percutaneous VSD intervention, and there are few studies on MITC against VSD. Tucker et al. [Tucker 2007] reported the results of a 20-year follow up of 4432 patients, who underwent surgical repair for VSD, including 48 cases of complete atrioventricular block implanted with permanent pacemakers $(1.1 \%)$. Twelve patients who had perimembranous VSD underwent periventricular closure by a minimally invasive incision without $\mathrm{CPB}$; all procedures were successful with no operative mortality, atrioventricular block, new aortic incompetence, or residual shunt [Zeng 2008]. New techniques and devices have revolutionized the transcatheter technique but still cannot achieve the surgical ability to close all types of atrial septal defect or VSD, control arrhythmias, and correct additional valve disease or malformation [Cho 2017]. This study showed that the incidence of early arrhythmias after MITC for VSD was $12.7 \%$, among which IRBBB

Table 3. Incidence of abnormal ECG at different time points s after MITC in 252 VSD patients [n, (\%)]

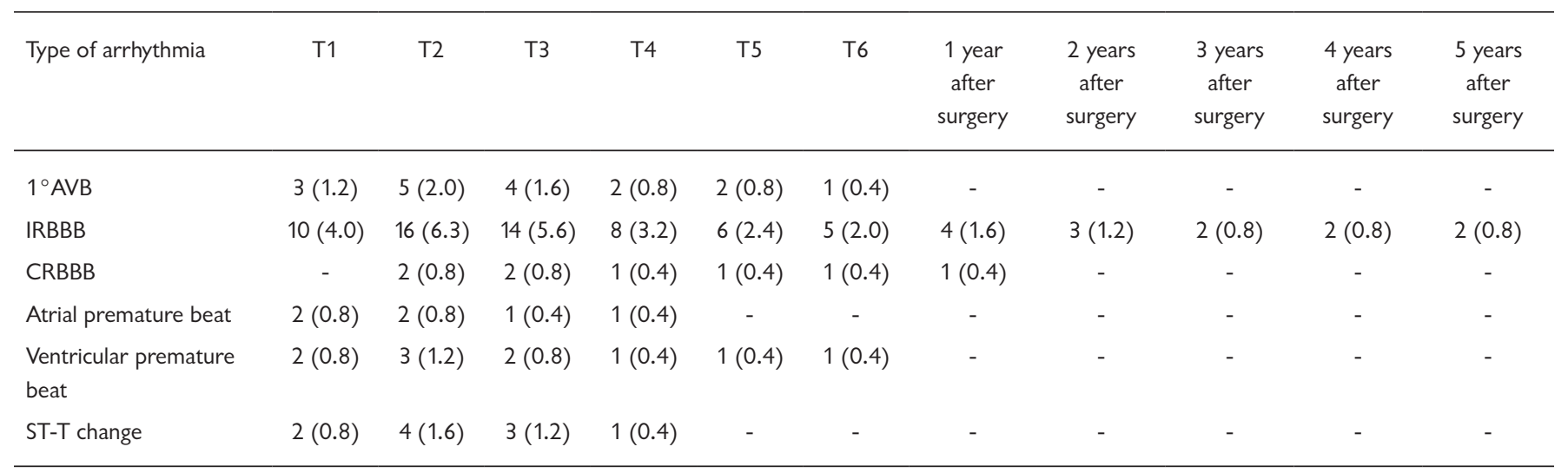

- refers to $N / A$

Table 4. Comparison of continuous variables between the patients with or without postoperative arrhythmia

\begin{tabular}{|c|c|c|c|c|}
\hline & Non-arrhythmia group & Arrhythmia group & $\mathrm{Z} / \mathrm{F}$ & $P$ \\
\hline Age (month) & $22.25 \pm 24.32$ & $26.97 \pm 29.67$ & -0.831 & 0.406 \\
\hline \multicolumn{5}{|l|}{ Gender } \\
\hline Male & 103 & 16 & 0.138 & 0.710 \\
\hline \multicolumn{5}{|l|}{ Defect site } \\
\hline Peripheral membrane & 183 & 29 & 1.619 & 0.655 \\
\hline Intra-crista & 16 & 2 & & \\
\hline Super-crista & 3 & 0 & & \\
\hline Sub-trunk & 20 & 1 & & \\
\hline VSD diameter (mm) & $3.37 \pm 1.43$ & $4.28 \pm 1.92$ & -2.871 & 0.004 \\
\hline Operation time (min) & $63.34 \pm 25.07$ & $77.83 \pm 23.37$ & 8.971 & 0.003 \\
\hline Occluder model (mm) & $5.36 \pm 1.28$ & $6.50 \pm 1.66$ & -4.104 & 0.000 \\
\hline \multicolumn{5}{|l|}{ Occluder type } \\
\hline Symmetrical & 191 & 30 & 4.777 & 0.029 \\
\hline Eccentric & 31 & 2 & & \\
\hline
\end{tabular}


Table 5. Binary multivariate logistic regression analysis of risk factors for arrhythmia after MITC

\begin{tabular}{|c|c|c|c|c|c|c|c|}
\hline VSD shunt (mm) & 0.408 & 0.149 & 7.482 & 0.006 & 1.503 & 1.123 & 2.013 \\
\hline Operation time (min) & 0.016 & 0.008 & 4.309 & 0.038 & 1.016 & 1.001 & 1.032 \\
\hline
\end{tabular}

and first-degree atrioventricular block were more common, and only two cases of CRBBB occurred. After 5 years of follow up, the cases eventually converted to IRBBB; this is considered to be relevant to insufficient experience, which led to the selection of a larger occluder. No serious arrhythmia or delayed arrhythmia occurred in this study, which shows that the incidence of arrhythmia after MITC is generally low. No serious arrhythmia occurred, considering that transthoracic occlusion does not require the establishment of a full delivery track across the tricuspid annulus through the vena cava and aorta, making the operation distance short with strong controllability [Cardiovascular Surgeons Branch of Chinese Medical Association 2011]. The sheath can enter the VSD from a basically vertical angle, thus avoiding repeated stimulation to the internal catheter of the heart cavity; at the same time, intraoperative esophageal ultrasound is used for fulltime, real-time, and dynamic evaluation of the size, shape, adjacency, and valve regurgitation of the VSD [Lee 2015; Cresti 2018].

The multivariate logistic regression analysis showed that the risk factors for postoperative arrhythmia were as follows: 1) VSD diameter: The larger the diameter of the VSD, the closer the conduction bundle is to the edge of the defect or even direct exposure. Therefore, the conduction bundle is more susceptible to intraoperative stimulation and to injury caused by the pressure of the occluder; at the same time, the larger the VSD, the greater the shunt in the heart cavity, the greater the impact on the hemodynamics, and the greater the likelihood of myocardial damage and secondary fibrosis of the heart endometrium at the edge of the VSD, which may form scars, involve the conduction system, and cause arrhythmia [Schlotter 2016]. 2) Occluder size: The larger the diameter of the occluder, the closer the outer edge of the occluder is to the conduction bundle, and the easier it is to directly compress the conduction bundle or cause higher tension at the edge of the VSD, thus causing edema around the defect and indirectly compressing the conduction bundle of the heart [Stonawski 2018]. 3) Presence of a membranous bulging tumor: The postoperative impact of membranous tumors in VSD on the incidence of arrhythmia is inconclusive. Szkutnik et al. [Szkutnik 2007] reported that the probability of postoperative arrhythmia caused by membranous tumors in VSD is significantly increased; however, Wu et al. [Wu 2009] believe there is no correlation between membranous tumors and postoperative arrhythmias. This study showed that the presence of membranous tumors is a protective factor against postoperative arrhythmias, and children without membranous tumors are more prone to experiencing arrhythmia after surgery. This may be attributed to the fact that when blocking VSD combined with membranous tumors, the left umbrella disk will be pulled into the tumor as much as possible to reduce the compression of the occluder on the surrounding tissue of the defect, thereby reducing the probability of arrhythmia.

On the ECG during follow up, the PR interval was prolonged at T1-3 and the QRS interval at T2 and T3. When the occluder touches, squeezes, and rubs the surrounding tissue, myocardial edema easily can occur, which then involves the conduction bundle and prolongs the PR interval and QRS interval; this is attributed to the close relationship between VSD and the conduction bundle. This also may explain why arrhythmia more often occurs in this period; thus, ECG indices should be monitored closely within 1 week after surgery. Owing to the large amount of left-to-right shunting in the heart cavity of patients with VSD, the left ventricle is enlarged and gradually thickened due to overcapacity. On EGC, the amplitudes of the Vl-lead S wave+V5-lead $\mathrm{R}$ wave and the electrical axis commonly are used to indirectly measure the left ventricular load and the changes in the degree of hypertrophy. Therefore, we opted to evaluate the Vl-lead S wave, V5-lead R wave, and left axis deviation. By comparison, the SV1+RV5 amplitude showed a significant downward trend since T6, and the left axis deviation recovered significantly at T6. The reason is that after closure, the ventricular horizontal shunt disappears, and the left ventricular end-diastolic diameter gradually decreases over time; thus, the heart load is reduced, and the compliance changes.

The reasons for the low incidence of arrhythmia included the following: 1) Unlike in traditional CPB and transcatheter VSD closure, there is no need to establish a complete delivery track across the tricuspid annulus through the vena cava and aorta and to suture under CPB in MITC. Herein, the sheath of the VSD was vertical to the VSD, avoiding repeated stimulation of the catheter. 2) This procedure is conducive to the choice of occluder size and type; thus, the selected occluder is often smaller than that in the transcatheter approach, which can minimize the friction and compression on the edge of the defect, especially the damage to the conduction bundle around the VSD. As such, the incidence of arrhythmia was low, and there was no serious arrhythmia. 3) Because of the 
careful, real-time, and dynamic evaluation of the size, shape, adjacent, and valve regurgitation of the VSD via ultrasound and the real-time observation of the ECG changes during surgery, we could select the appropriate occlude, according to the size and shape of the VSD to achieve individualized treatment; therefore, no serious arrhythmia occurred in this study.

In summary, we believe that MITC for VSD is safe and reliable. Although the incidence of postoperative arrhythmia is low, the following points should be achieved to avoid myocardial damage as much as possible: 1 ) The surgeon must have abundant knowledge of cardiac anatomy and cardiac ultrasound, and the sonographer must have extensive experience of cardiac ultrasound examination and can cooperate with the surgeon in tacit understanding, so that the most suitable occluder can be selected from professional perspectives to avoid closure injury to the surrounding tissues. 2) Surgical indications should be strictly followed, and ultrasound findings should carefully be checked before surgery. For children with large VSD around the membrane without membranous tumors, closure should not be performed under any risk, which will easily increase the risk of arrhythmia. 3) Intraoperative esophageal ultrasound should be performed to explore the position and size of VSD in multiple aspects for selecting the appropriate occluder as much as possible; meanwhile, the number of intraoperative occluder replacements should be reduced. 4) The surgical skills should be standardized; the movements should be gentle; and the operation time should be reduced, thus avoiding repeated stimulation to the edge of the VSD and causing myocardial edema. 5) Postoperative ECG changes should be closely monitored, and regular reviews should be performed to facilitate early detection and treatment.

MITC technology has developed rapidly in recent years and routinely has been used in many Chinese heart centers; however, long-term follow-up data still are lacking. This study objectively explored the long-term efficacy of this procedure and the related prognosis based on ECG indices and provided objective data for actual follow up and evaluation and strong evidence for the safety of this technology.

\section{ACKNOWLEDGEMENTS}

This study was supported by the Natural Science Foundation of Liaoning Province (20180550623) and the projects of Dalian Science and Technology Plan Foundation (2015E12SF127).

\section{REFERENCES}

Cardiovascular Surgeons Branch of Chinese Medical Association. 2011. Chinese Expert Consensus of Transthoracic Minimally Invasive closure for Ventricular Defect. Chinese Journal of Thoracic and Cardiovascular Surgery 27:516-8.

Cho YS, Park SE, Hong SK, Jeong NY, Choi EY. 2017. The natural history of fetal diagnosed isolated ventricular septal defect. Prenat Diagn 37:889-93.
Cresti A, Giordano R, Koestenberger M, et al. 2018. Incidence and natural history of neonatal isolated ventricular septal defects: do we know everything? A 6 year single center Italian experience follow-up. Congenit Heart Dis 13:105-12.

Faccini A, Butera G. 2019. Techniques, Timing, and Prognosis of Transcatheter Post Myocardial Infarction Ventricular Septal Defect Repair. Curr Cardiol Rep 21:59.

Gerbode F, Hultgren H, Melrose D, Osborn J. 1958. Syndrome of left ventricular-right atrial shunt. Successful surgical repair of defect in five cases, with observation of bradycardia on closure. Ann Surg 148:433-46.

Habib G, Lancellotti P, Antunes MJ, et al. 2015. 2015 ESC guidelines for the management of infective endocarditis: the task force for the Management of Infective Endocarditis of the European Society of Cardiology (ESC) Endorsed by: European Association for Cardio Thoracic Surgery (EACTS), the European Association of Nuclear Medicine (EANM). Eur Heart J 36:3075-128.

Lee WC, Fang CY, Huang CF, Lin YJ, Wu CJ, Fang HY. 2015. Predictors of atrial septal defect occlude dislodgement. Int Heart J 56:428-31.

Li X, Song GX, Wu LJ, et al. 2016. Prediction of spontaneous closure of isolated ventricular septal defects in utero and postnatal life. BMC Pediatr 16:207.

Liang F, Li HX, Zhang HZ, Guo WB, Zou CW, Farhaj Z. 2017. Perventricular double-device closure of wide-spaced multi-hole perimembranous ventricular septal defect. J Cardiothorac Surg 12:24.

Ou-yang WB, Wang SZ, Hu SS, Zhan FW, Zhang DW, et al. 2017. Perventricular device closure of perimembranous ventricular septal defect: effectiveness of symmetric and asymmetric occluders. Eur J Cardiothorac Surg 51:478-82.

Penny DJ, Vick GW III. 2011. Ventricular septal defect. Lancet 377:1103-12.

Rein JG, Freed MD, Norwood WI, Castaneda AR. 1977. Early and late results of closure of ventricular septal defect in infancy. Ann Thorac Surg 24:19-27.

Schlotter F, de Waha S, Eitel I, Desch S, Fuernau G, Thiele H. 2016. Interventional post-myocardial infarction ventricular septal defect closure: a systematic review of current evidence. EuroIntervention 12:94-102.

Soto B, Becker AE, Moulaert AJ, Lie JT, Anderson RH. 1980. Classification of ventricular septal defects. Br Heart J 43:332-43.

Spicer DE, Anderson RH, Backer CL. 2013. Clarifying the surgical morphology of inlet ventricular septal defects. Ann Thor Surg 95:236-41.

Spicer DE, Hsu HH, Co-Vu J, Anderson RH, Fricker FJ. 2014. Ventricular Septal Defect. Orphanet J Rare Dis 9:144.

Stonawski V, Vollmer L, Kohler-Jonas N, et al. 2018. Long-term associations of an early corrected ventricular septal defect and stress Systems of Child and Mother at primary school age. Front Pediatr 5:293.

Szkutnik M, Qureshi SA, Kusa J, Rosenthal E, Bialkowski J. 2007. Use of the Amplatzer muscular ventricular septal defect occluder for closure of perimembranous ventricular septal defects. Heart 93:355-8.

Tucker EM, Pyles LA, Bass JL, Moller JH. 2007. Permanent pacemaker for atrioventricular conduction block after operative repair of perimembranous ventricular septal defect. J Am Coll Cardiol 50:1196-200.

Wu H, Qin Y, Zhao XX, et al. 2009. Transcatheter closure of multihole perimembranous VSD with aneurysm: 3-year follow-up study. Clin Res Cardiol 98:563-9. 
Xing QS, Pan SL, Zhuang ZY, et al. 2009. Minimally invasive perventricular device closure of an isolated perimembranous ventricular septal defect with a newly designed delivery system: preliminary experience. J Thorac Cardiovasc Surg 137:556-9.

Zeng XJ, Sun SQ, Chen XF, et al. 2008. Device closure of perimembranous ventricular septal defects with a minimally invasive technique in 12 patients. Ann Thorac Surg 85:192-4.

Zhang GC, Chen Q, Cao H, Chen LW, Yang LP, Chen DZ. 2013. Minimally invasive perventricular device closure of ventricular septal defect in infants under transthoracic echocardiograhic guidance: feasibility and comparison with transesophageal echocardiography. Cardiovasc Ultrasound 11:8. 